\title{
Modulation of angiogenesis by inflammatory markers and the role of matrix metalloproteinases in an endothelial cell/fibroblast co-culture system.
}

BARRON, G.A., BORDET, E., GOUA, M. and BERMANO. G. 


\title{
Modulation of Angiogenesis by Inflammatory Markers and the Role of Matrix Metalloproteinases in an Endothelial Cell/Fibroblast Co-culture System
}

\author{
Gemma A. Barron, Elise Bordet, Marie Goua and Giovanna Bermano* \\ Institute for Health and Wellbeing Research, Robert Gordon University, Riverside East, Garthdee Road, \\ Aberdeen, Scotland, AB10 7GJ, United Kingdom
}

\begin{abstract}
Increased levels of inflammatory markers such as tumour necrosis factor- $\alpha$ (TNF $\alpha)$ and inter- leukin- 6 (IL-6) have been associated with formation of new blood vessels, or angiogenesis, and linked to chronic inflammation in obesity. This study aimed to establish and use a versatile co-culture cell system to further investigate the role of TNF $\alpha$ and IL-6 in modulating (i) tubule formation and (ii) cell-cell interactions via matrix metalloproteinase (MMP) enzyme activity and secretion of vascular endothelial growth factor (VEGF), E-selectin and prostaglandin $\mathrm{E}_{2}\left(\mathrm{PGE}_{2}\right)$. Co-cultures of human endothelial cells and fibroblasts were incubated with $\mathrm{TNF} \alpha$ $(10 \mathrm{ng} / \mathrm{mL})$ or IL-6 $(10 \mathrm{ng} / \mathrm{mL})$ added 2 and/or 7 days after co-culture establishment. Cell viability by enzymatic conversion was determined by MTT assay; tubule formation was detected by immunostaining; VEGF, E-selectin and PGE $_{2}$ expression by ELISA analysis and MMP enzyme activity by gel zymography. Treatment- specific and time dependent differences in tubule formation were observed: IL-6 significantly increased tubule formation, whilst TNF $\alpha$ significantly inhibited tubule formation. Treatment-specific differences in levels of MMP activities which correlate to tubule formation were also observed. This study showed inflammatory markers, typically associated with obese status, affect tubule formation differently in a heterogeneous cell environment similar to that observed in vivo.
\end{abstract}

Keywords: Angiogenesis, co-culture, cytokines, fibroblasts, HUVEC, obesity.

\section{INTRODUCTION}

Inflammatory markers such as tumour necrosis factor- $\alpha$ (TNF $\alpha$ ) [1], interleukin-6 (IL-6) [2], along with leptin [2] and hepatocyte growth factor (HGF) [3] have been shown to be elevated in plasma of obese individuals [4] and considered a major risk factor for the development of long term chronic conditions (e.g., cardiovascular disease, type 2 diabetes, stroke and cancer) [5,6] and vascular complications [7]. As some of these inflammatory markers have been implicated in the increased development of angiogenesis [8-11], it is very important to identify and characterise the role that such inflammatory markers have on modulating angio- genesis.

Angiogenesis, or the growth of new blood vessels, is an important multi-step process, which occurs both in the maintenance of health and in certain disease states [12]. It plays a key role in normal vascular tissue development and repair (e.g., natural wound healing), but, when disease occurs, it can be deregulated and end in an abnormal increase of vessel density (e.g., tumourgenesis, diabetic retinopathy and atherosclerotic plaques) [13]. Additionally, angiogenesis plays a crucial role in the modulation of adipogenesis [14].

Angiogenesis involves an extensive interplay between a variety of growth factors, cytokines, enzymes and cells, together with extracellular matrix (ECM) components $[13,15,16]$. Vascular endothelial growth factor (VEGF) is a major angiogenic growth factor that stimulates proliferation and migration of endothelial cells (EC) [17] and is secreted by almost all solid tumours, tissue-infiltrating macrophages and host stromal cells [18]. Other important growth factors are basic fibroblast growth factor (b-FGF), HGF and angiopoietin (Ang)-1 and 2 [13]. These growth factors exert their biological activity on EC through various signalling pathways; where excess growth factors stimulate angiogenesis. Interestingly, many of the cytokines and growth factors responsible for angiogenesis are also regulators of tissue- degrading matrix metalloproteinase (MMP) enzymes [19].

MMP enzymes are a family of structurally related proteinases which are readily able to degrade all components of the ECM [20,21]. The regulation of MMP activity is known to play a key role in EC migration during angiogenesis [22]. There is little or no basal production of MMP enzymes in most cell types. However, MMP expression and activity are inducible: with regulators known to include growth factors, cytokines and cell-matrix interactions, and their role in cancer has been implicated in tumour invasion and metastasis [21]. More specifically, MMP-2 (Gelatinase A, $72 \mathrm{kDa}$ ) and MMP-9 (Gelatinase B, $92 \mathrm{kDa}$ ), which degrade denatured collagens and basement membrane components, have been associated with this process [21]. MMP-2 is typically produced in most human tissue cell types and, interestingly, elevated levels of MMP-2 are associated with invasive behaviour [23].

*Address correspondence to this author at the Institute for Health and Well- being Research, Robert Gordon University, Riverside East, Garthdee Road, Aberdeen, Scotland, AB10 7GJ, United Kingdom; Tel: +44 1224 262885; Fax: +44 1224 262828; E-mail: g.bermano@rgu.ac.uk 
Several in vitro and in vivo methods have been established over the years to study the factors that influence angiogenesis. These include monoculture assays (such as motility assays, matrigel formation or proliferation assays) or organ and tissuespecific assays (such as cornea assay or the chick chorioallantoic membrane (CAM) assay) [24]. However, the co-culture assay system established by Bishop and colleagues [15], in which primary human EC and primary normal human dermal fibroblasts are grown together, overcomes the limitations of the former assays and, in particular, can be used to observe the generation and growth of endothelial tubules, as well as inhibition, within a heterogeneous cellular environment [24]. In this study, the co-culture system established by Bishop et al. [15] has been further developed to create a more versatile system which allows the additional study of the correlation between specific inflammatory markers and vessel formation and progression.

The present study aimed to use this more versatile in vitro co-culture system to determine the role of TNF $\alpha$ and IL- 6 two highly elevated pro-inflammatory cytokines, typically associated to the obese status - in modulating (i) tubule formation and (ii) cell-cell interactions via MMP enzyme activity and secretion of VEGF, E-selectin and PGE2.

Using the versatile co-culture assay model, treatment- specific and time-dependent differences in tubule formation were observed; IL-6 significantly increased tubule formation, whilst TNF $\alpha$ significantly inhibited it. Moreover, treatment specific differences in levels of MMP enzyme activities - which influence tubule formation - were observed. These results add further knowledge to the understanding of the role of inflammatory markers, commonly associated to the obese status, in modulating angiogenesis.

\section{MATERIALS AND METHODS}

\section{Materials}

All chemicals and cell culture reagents were purchased from Sigma-Aldrich Ltd (UK) unless otherwise stated.

\section{Cell Culture}

Primary human umbilical vein endothelial cells (HUVEC) and primary normal human dermal fibroblasts (NHDF) were obtained from Clonetics (Lonza, UK). HUVEC were maintained in endothelial basal medium (EBM-2; Clonetics, Lonza, UK) supplemented with EGM-2 SingleQuots (Clonetics, Lonza, UK) (i.e., EGM-2 medium) whilst NHDF were maintained in fibroblast basal medium (FBM; Clonetics, Lonza, UK) supplemented with FGM-2 Single- Quots (Clonetics, Lonza, UK) (i.e., FGM-2 medium).

The EC/fibroblast co-culture system was set up using HUVEC (between passages 1-8) and NHDF (between passages 1-12) following the protocol established by Bishop and colleagues [15]. HUVEC and NHDF were mixed and seeded in 24 well plates (Thermo Fisher Scientific Nunc, UK) in EGM-2 medium. Co-cultured cells were incubated for up to 14 days at $37^{\circ} \mathrm{C}$ in a $5 \% \mathrm{CO}_{2}$ in air humidified incubator.

The $₫$-culture system was manipulated and tested by removing VEGF from the EGM-2 medium (EGM-2Ө: EGM-2 medium minus the SingleQuot of VEGF) and by adding exogenous VEGF $(50 \mathrm{ng} / \mathrm{mL})$ and/or anti- VEGF (50 ng/mL).

\section{Cell Viability by Enzymatic Conversion}

HUVEC and NHDF $\left(1 \times 10^{5}\right.$ cells/mL) were incubated with different concentrations of TNF $\alpha$ or IL-6 (100 $\mu$ L/well; 96 hours) in EGM-2 and FGM-2 medium, respectively. Cell viability was evaluated by MTT ((3-(4, 5-dimethyl-thiazol-2yl)-2, 5-diphenyltetrazolium bromide) assay [25]. Absorbance was read at $560 \mathrm{~nm}$ in a plate reader (Bio-Tek, UK).

\section{Co-culture Treatment}

The co-culture system was incubated with EGM-2 medium to initiate tubule formation and, 2 and/or 7 days after coculture establishment, medium was changed to EGM-2Ө (i.e., EGM-2 medium minus VEGF SingleQuot), EGM-2 $\Theta$

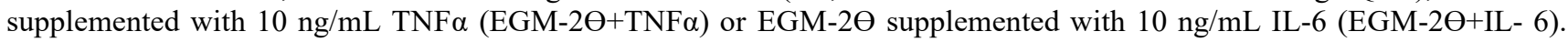
The medium was collected every 4 days and replaced with respective treatments. Collected medium was stored at $-20^{\circ} \mathrm{C}$ until use.

The expression of secreted VEGF (DuoSetELISA, R\&D Systems, Cat\# DY293B), human E-selectin/CD62E (DuoSet ELISA, R\&D Systems, Cat\# DY724) and human prosta- glandin $\mathrm{E}_{2}$ ( $\mathrm{PGE}_{2}$ : Parameter ELISA, R\&D Systems, Cat\# KGE0004B) in the collected media were measured according to manufacturer' instructions. These assays have a sensitivity of approximately $30 \mathrm{pg} / \mathrm{mL}$ and quantify both the natural and recombinant forms of each protein.

\section{Immunostaining}

After 14 days, co-cultures were fixed at room temperature with ice-cold $70 \%(\mathrm{v} / \mathrm{v})$ ethanol and stained with myeloma cell adhesion molecule (MCAM (CD146); specific to HUVEC) or collagen type IV to assess the extent of tubule formation. MCAM allowed the visualisation of tubule formation and growth and was detected with monoclonal mouse anti-human MCAM antibody (R\&D Systems, USA) with the secondary goat anti-mouse IgG (whole antibody) conjugated with alkaline phosphatase. Sigma FAST 5-bromo-4-chloro- 3-indolyl phosphate/nitro blue tetrazolium (BCIP/NBT) was the substrate with the reaction yielding a dark purple end product. The co-cultures were dried overnight at room temperature 
and analysed. Collagen type IV was used to visualise the presence of lumen. A monoclonal mouse anti-human collagen type IV antibody was used with the same secondary and substrate as before.

Co-cultures were viewed using an Olympus 1XS1 microscope with Olympus TL4 light box. The image software used was Infinity Capture Application Version 5.0.0 (Lumenera Corporation, Canada) with camera model Infinity 2-2c. Three images per well were captured and saved as BMP images of 1616 x 1216 pixels. Illumination was set to give a best contrast between stained tubules and unstained fibro- blasts. Tubule formation was defined by total tubule length and quantification was achieved by AngioSys ${ }^{\circledR}$ software Version 1.0 (TCS Cell Works, UK).

\section{Gel Zymography}

For the analysis of MMP-2 and MMP-9 enzyme activity, the medium was collected from 3 separate experiments and quantification of the total protein secreted carried out by the Bradford protein assay, according to manufacturer's instructions. MMP enzyme activity was assessed in relation to tubule formation at the end of co-culture incubation by gel zymography adapted from Connelly and colleagues [22]. Briefly, equal volumes of samples were loaded onto a 10\% SDSpolyacrylamide gel containing $0.1 \%(\mathrm{v} / \mathrm{v})$ porcine gelatin. After electrophoresis, to remove the SDS, the gels were washed twice for 15 minutes each in $2.5 \%$ (v/v) Triton $\mathrm{X}-100$ and then incubated overnight at $37^{\circ} \mathrm{C}$ in substrate buffer $(50 \mathrm{mM}$ Tris-HCl, pH 7.6 and $10 \mathrm{mM} \mathrm{CaCl}_{2}$ ) in the absence and presence of $10 \mathrm{mM}$ EDTA. Gels were stained for 40 minutes with two changes of $0.5 \%$ Coomassie Blue R-250 (ICN Biomedical, USA), destained twice for 20 minutes in 50\% (v/v) methanol, $10 \%(\mathrm{v} / \mathrm{v})$ acetic acid and dried (Dry Ease Mini Gel drying system, Invitrogen, UK). MMP enzymes were visualised with areas of gelatinolytic activity which appear to be clear against the dark background of the stained gel. MMP enzymes were analysed using Image J 1.43u software (NIH, USA) and a Kaleidoscope Precision Plus Protein Standard (Bio-Rad, UK) was used to determine the molecular weight of each MMP band.

\section{Statistical Analysis}

Unless otherwise stated, all data are expressed as mean \pm SEM of three independent experiments from different cell passage numbers. Statistical differences were determined by unpaired $t$-test (Graphpad Prism) or by one-way analysis of variance (ANOVA) with Dunnett's multiple comparison $t$-test (Graphpad Prism). $P<0.05$ was considered to be statistically significant.

\section{RESULTS}

\section{Effect of TNFa or IL-6 on Cell Viability}

Cell viability in HUVEC and NHDF was quantified by MTT assay where mitochondrial activity is assumed to reflect the rate of cell metabolism and, thus, the rate of enzymatic conversion is directly representative of cell viability. The cells were incubated with different concentrations $(0.01-200 \mathrm{ng} / \mathrm{mL})$ of TNF $\alpha$ or IL-6 for 96 hours (Fig. 1). The percentage cell viability was normalised to the corresponding untreated control (i.e., PBS for TNF $\alpha$ or $\mathrm{H}_{2} \mathrm{O}$ for IL-6).

No change to cell viability, compared to the control, was observed between $0.01-10 \mathrm{ng} / \mathrm{mL}$ TNF $\alpha$ in HUVEC, but cell viability was significantly decreased with 100 and $200 \mathrm{ng} / \mathrm{mL} \mathrm{TNF} \alpha$; by 25 and 18\%, respectively (Fig. 1A). In- creased cell viability, compared to the control, was observed between 0.01-1 ng/mL TNF $\alpha$ in NHDF with a significant increase of 119, 112 and 107\% with 10, 100 and $200 \mathrm{ng} / \mathrm{mL}$ TNF $\alpha$, respectively (Fig. 1B). No change to cell viability, compared to the control, was observed with 0.01-200 ng/mL IL-6 in either HUVEC (Fig. 1A) or NHDF (Fig. 1B). Thus, $10 \mathrm{ng} / \mathrm{mL}$ was chosen for both cytokines to be used for tubule formation experiments.

\section{Versatility of the Co-Culture System Used}

MCAM and collagen type IV staining were used throughout this study to assess tubule formation and growth (Fig. 2A), together with quantification of total tubule length using AngioSys software analysis. The formation of tubules mimicking the process of angiogenesis was observed, over 14 days, and structures resembling a microvasculature bed developed (Fig. 2B). The co-culture system was manipulated to test its dependence from the content of VEGF in the medium by incubating the co-culture in EGM-2 $\Theta$ and either adding exogenous VEGF (50 ng/mL) and/or anti-VEGF (50 ng/mL) (Fig. 2C). The extent of tubule formation observed in the different culture conditions (Fig. 2) highlights the versatility and usability of this system. Tubule formation obtained from cells co-cultured in EGM-2 medium was chosen as a positive control throughout this study. 
A.

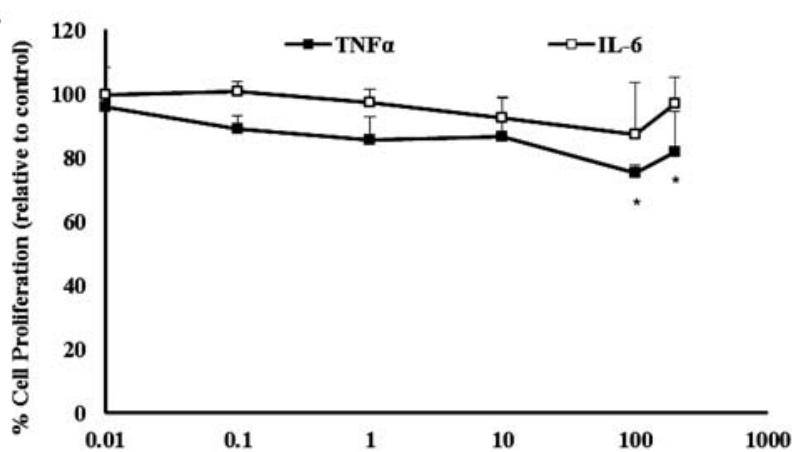

B.

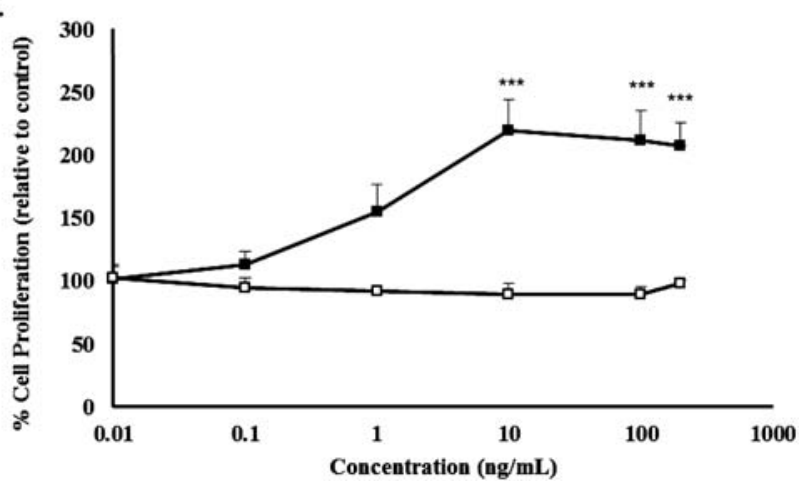

Fig. (1). Effect of TNF $\alpha$ or IL-6 on (A) HUVEC and (B) NHDF viability. Cell viability was determined using MTT assay after 96 hours. Data are mean \pm SEM of 3 independent experiments; each experiment consisted of 4 replicates per concentration; $* P<0.05$, $* * P<0.01$, $* * * P<0.001$. Note changes to the $\%$ cell viability scale (i.e., y axis).

\section{Effect of TNFa or IL-6 on Tubule Formation}

The effect of TNF $\alpha$ or IL-6 on tubule formation was tested with EGM-2 $\Theta$ medium in order to examine tubule formation in the absence of VEGF. Co-cultures were initially incubated in EGM-2 medium (Day 0) and, after establishment, EGM$2 \Theta$, EGM-2Ө+TNF $\alpha$ or EGM-2Ө+IL-6 were added after 2 and/or 7 days (Fig. 3). These conditions were used to assess the ability of these cytokines to respectively (i) promote/prevent tubule formation, or (ii) increase/reduce tubule formation once the process has already started.

Tubules were clearly formed and visualised after 14 days (Fig. 3A) and similar results were obtained in each experiment. The extent of tubule formation was quantified by measuring total tubule length and percentage change was normalised to the control (i.e., EGM-2Ө) (Fig. 3B). Tubule formation was significantly increased (43\% increase) in EGM-2 medium incubated co-cultures compared to control co-cultures. TNF $\alpha$ significantly prevented tubule formation by $95 \%$ when added on day 2 and significantly reduced tubule formation by $59 \%$ when added on day 7 compared to control co-cultures. Incubating co-cultures with IL-6 significantly promoted tubule formation by $46 \%$ when added on day 2 compared to control co-cultures. Whilst tubule formation was further increased by $57 \%$ when added on day 7 compared to control co-cultures, this was not statistically significant.

These results indicate TNF $\alpha$ and IL-6 altered several in vitro cell activities that are relevant to angiogenesis as expected, including cell viability and tubule formation. 
A.

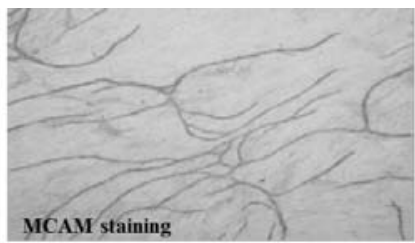

B.
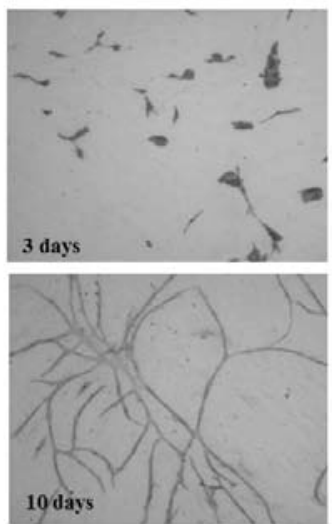

C.
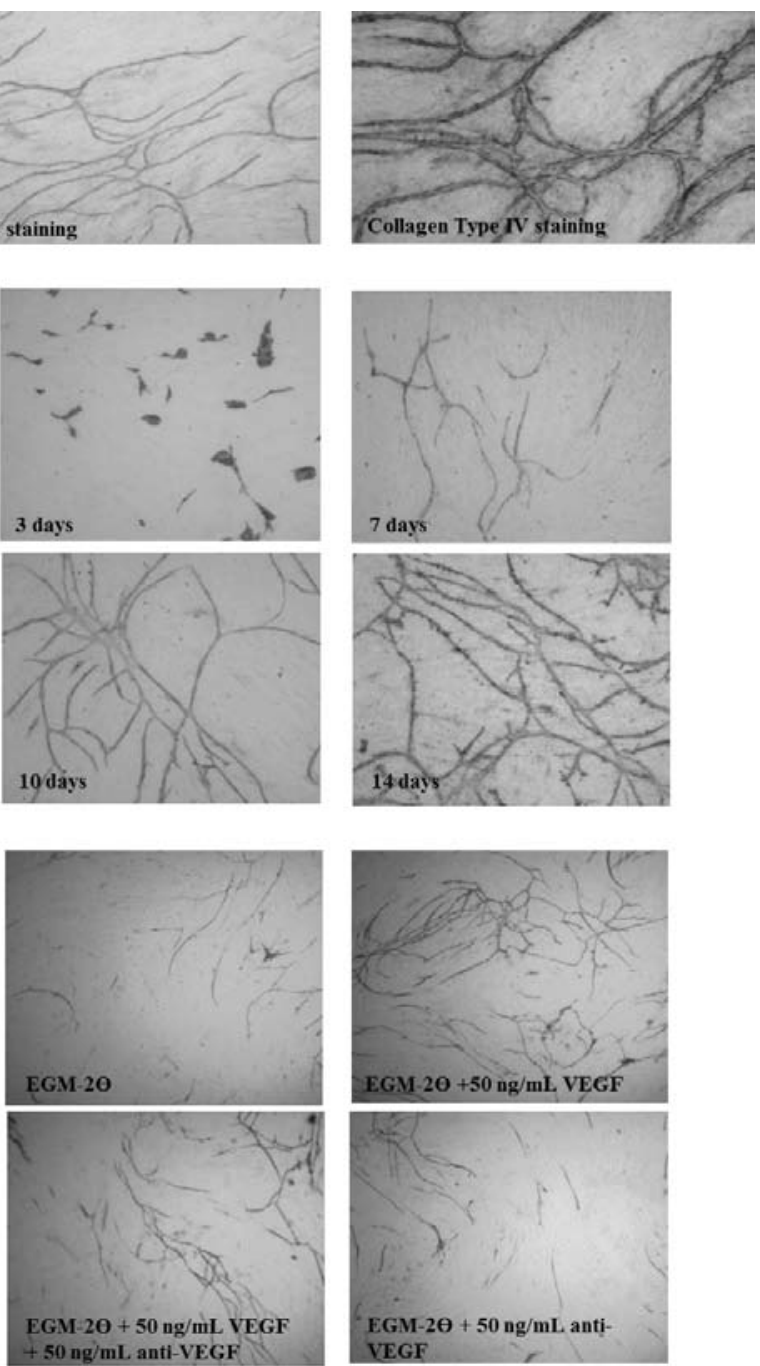

Fig. (2). (A) Tubule formation and growth assessed with MCAM and Collagen type IV staining of a typical co-culture on day 14. (B) Tubule formation over time (3 - 14 days) assessed by MCAM staining. (C) Tubule formation and growth assessed with MCAM staining at day 14 in

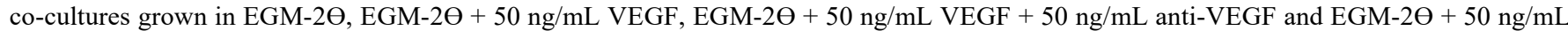
anti-VEGF added on day 0 . Medium was changed every 4 days. Each image is representative of 3 independent experiments; within an experiment, each treatment was carried out in duplicate and observed in three randomly chosen fields. Magnification 40x. 
A.
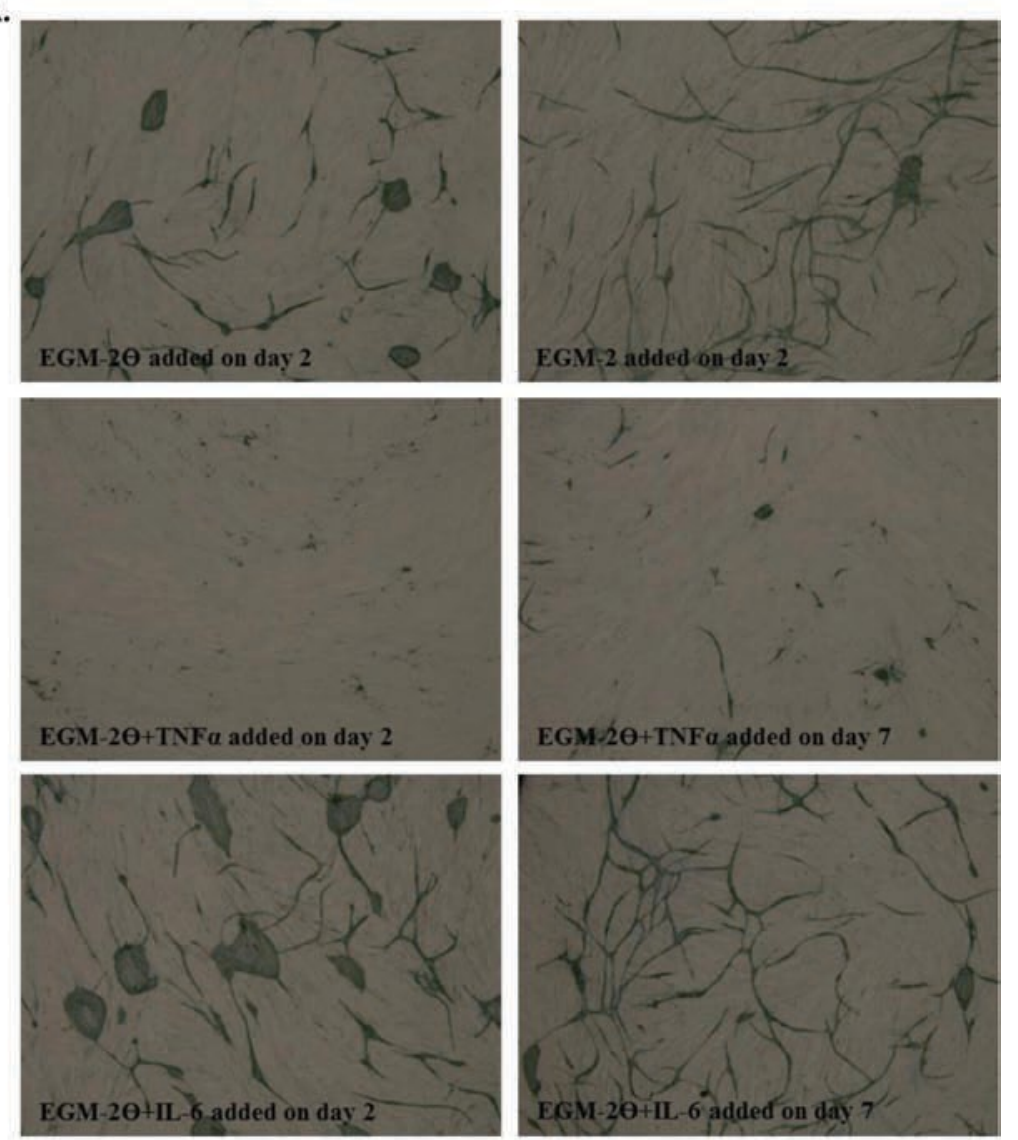

B.

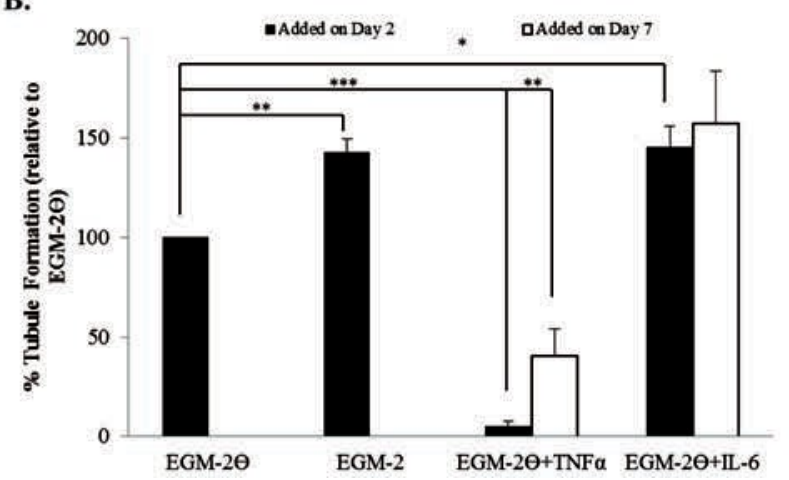

Fig. (3). (A) Tubule formation and growth assessed with MCAM staining at day 14 in co-cultures grown in EGM-2 $\Theta$ and EGM-2 (added

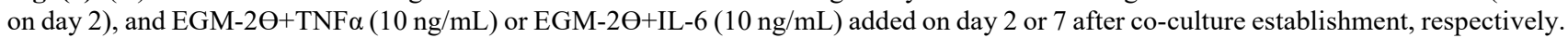
Medium was changed every 4 days. Images presented are representative of treatments from 3 independent experiments; within an experiment, each treatment was carried out in duplicate and observed in three randomly chosen fields. Magnification 40x. (B) Quantification of total tubule length determined using AngioSys software. Cytokines added on day 2 (black bars) and day 7 (white bars). Results are presented as a percentage of EGM-2 $\Theta$ as mean \pm SEM of 3 independent experiments; within each experiment, each treatment was carried out in duplicate; $* P<0.05, * * P<0.01, * * * P<0.001$.

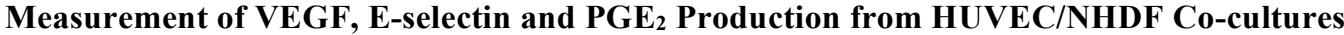

The effect of TNF $\alpha$ or IL- 6 in vitro was evaluated in rela- tion to the production of the well-characterised angiogenic factor and signalling factor for malignant progression, VEGF, of the adhesion molecule E-selectin and of the protumourigenic prostanoid $\mathrm{PGE}_{2}$ which are markers of inflammation (Fig. 4). VEGF, E-selectin and $\mathrm{PGE}_{2}$ levels were measured by ELISA, in the medium collected from HUVEC/NHDF co-cultures cultured over 14 days, in EGM-2

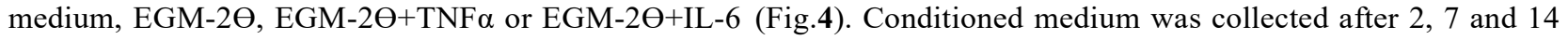

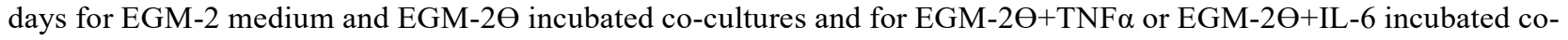
cultures on days 7 and 14. Results are presented as percentage change from the control (i.e., EGM-2Ө) on day 14. 
Firstly, measurement of VEGF levels revealed no significant changes in medium collected on day 14, from co- cultures incubated with EGM-2 medium or EGM-2Ө+IL-6 compared to control (Fig. 4A). This result suggests that VEGF is utilised for tubule development in this co-culture system and does not accumulate in the conditioned medium. Medium collected from EGM-2Ө+TNF $\alpha$ incubated co- cultures contained higher VEGF levels ( $50 \%$ and $72 \%$ increase when TNF $\alpha$ was added after 2 and 7 days, respectively) than in the control. A statistically significant increase in VEGF was observed when TNF $\alpha$ was added on day 7 (and collected on day 14) (Fig. 4A). The increased levels of VEGF in medium collected from TNF $\alpha$ treated co-cultures mirror the decrease in total tubule length (Fig. 3B). Secondly, results obtained from E-selectin measurement show no significant changes in E-selectin expression, except for a significant increase (508\%) in E-selectin expression with TNF $\alpha$ on day 7 (added on day 2) compared to control (Fig. 4B). Finally, measurement of PGE 2 shows that there were no significant effects on $\mathrm{PGE}_{2}$ secretion, irrespective of treatment, when compared to control, except for when co- cultures were incubated with EGM-2 medium (20\% de- crease) or EGM-2Ө+IL-6 (24\% decrease) added on day 2 (and collected on day 14) (Fig. 4C).

A.

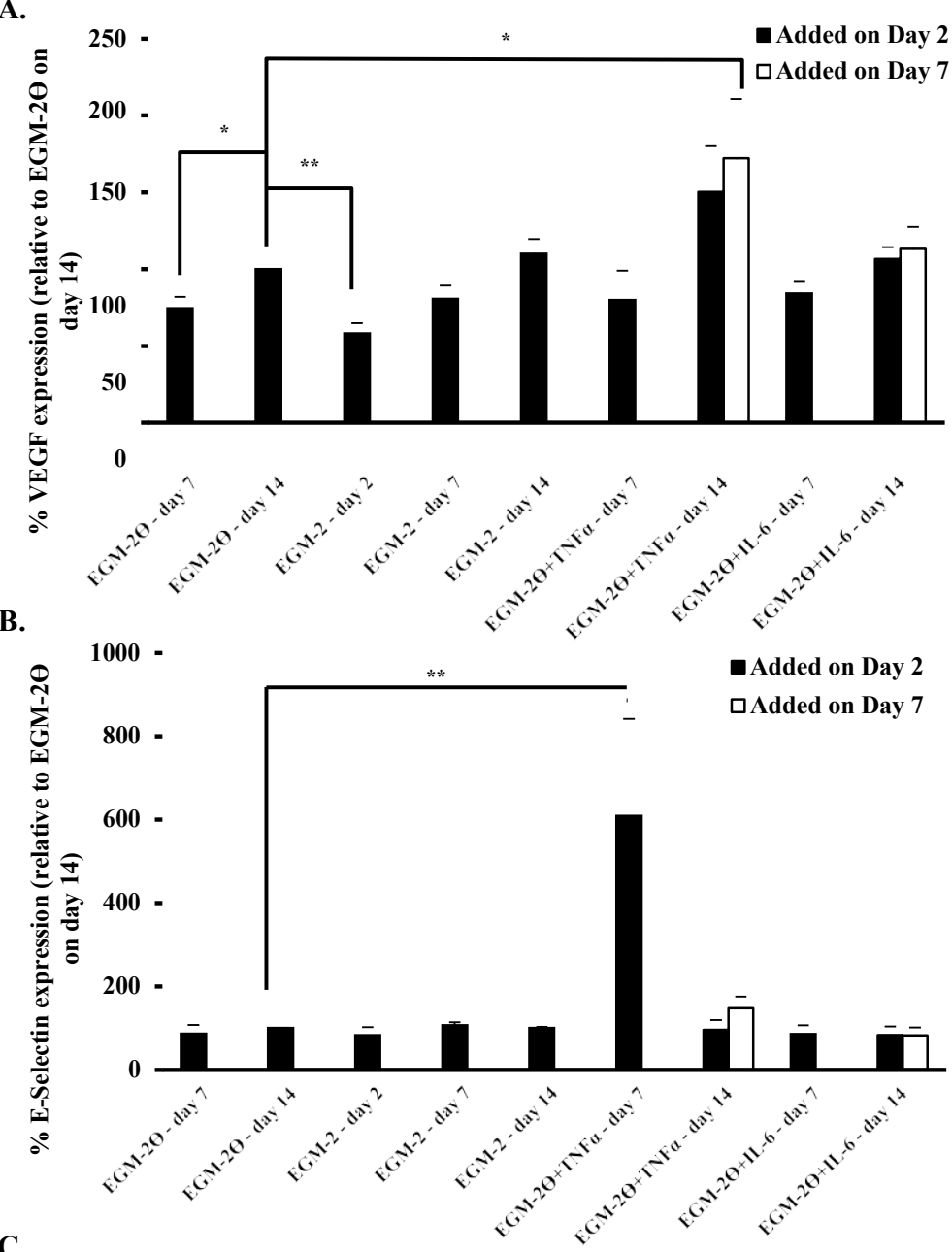

C.

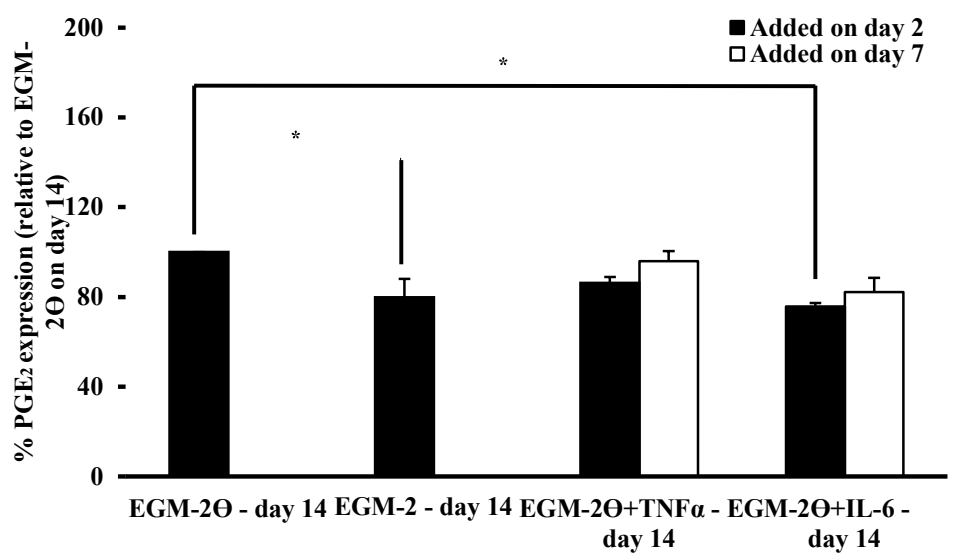

Fig. (4). Changes in (A) VEGF expression (\%) detected by a DuoSet human ELISA kit, (B) E-selectin expression (\%) detected by a DuoSet human ELISA kit, and (C) $\mathrm{PGE}_{2}$ expression (\%) detected by a Parameter human ELISA kit in the medium of HUVEC/NHDF co-cultures

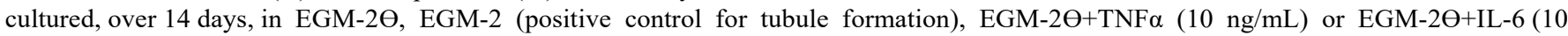
$\mathrm{ng} / \mathrm{mL}$ ). Results are presented as a percentage of EGM-2 $\Theta$ as mean \pm SEM of 3 independent experiments; within each experiment, each treatment was carried out in duplicate; $* P<0.05, * * P<0.01$. Note the changes to the $\%$ expression scale (i.e., y axis). 


\section{Effect of TNFa or IL-6 on MMP Enzyme Activity}

To analyse if modulation of angiogenesis by TNF $\alpha$ or IL-6 was associated to changes in the enzyme activity of proteases involved in matrix remodelling, MMP-2 and MMP-9 enzyme activity were measured by gel zymography. Medium from HUVEC and NHDF grown individually or in combination, as HUVEC/NHDF co-cultures, was assayed as described in Materials and Methods. The activity of MMP enzymes was visualised as areas of gelatinolytic enzyme activity which appear clear against the dark background of Coomassie Blue stained gels. Gels were analysed using Image J software and the molecular weight of each MMP band determined; $92 \mathrm{kDa}$ for MMP-9, $72 \mathrm{kDa}$ for pro-MMP-2 (inactive pro-enzyme) and $62 \mathrm{kDa}$ for active-MMP-2 (cleaved, activated enzyme). No bands were detected in control gels incubated in the presence of EDTA (data not shown).

The activity of MMP enzymes was initially determined in the medium of HUVEC and NHDF individually (Fig. 5). Cell culture medium was collected on days 2, 7, 10 and 14 after culture establishment and results show only pro-MMP-2 (inactive form) (Fig. 5A). Pro-MMP-2 activity was quantified and a statistically significant increase in pro-MMP-2 activity was observed in a time-dependent manner in both NHDF and HUVEC. Activity increased by 5- and 8-fold at day 7 and 10 of co-culture, respectively, in NHDF compared to activity at day 2. No further statistically different increase was observed at day 14. Similarly, activity increased by 8 - and 11 -fold respectively, in HUVEC compared to activity on day 2 , with no further increase observed at day 14.

MMP-9 and active-MMP-2 were not detected in NHDF and HUVEC when cultured individually (Fig. 5), however, in co-culture (medium collected at final end point - day 14), these MMP enzymes could be detected using gel zymography (Fig. 6A). The results show pro-MMP-2 activity was higher than active-MMP-2 activity when tubules were formed in control (i.e., EGM-2Ө) incubated co-cultures and in co-cultures grown with EGM-2 medium (statistically significant difference, $P<0.05$ ) or EGM-2Ө+IL-6 (Fig. 6B and C), with the exception of co-cultures incubated with EGM-2 $\mathrm{C}+\mathrm{TNF} \alpha$ on day 2 and 7 when tubules were not formed (statistically significant difference $(P<0.05)$ when TNF $\alpha$ added on day 7 , but not when added on day 2) (Fig. 6B and C). Similar levels of pro-MMP-2 were observed irrespective of treatment. ActiveMMP-2 activity increased in EGM-2Ө+TNF $\alpha$ treated co-cultures (no tubules) compared to control co-cultures but this was not statistically significant. This trend was similar for treatments added on day 2 or day 7 . No MMP-9 activity was found in any of these samples. However, in TNF $\alpha$ treated co-cultures, significantly higher levels of MMP-2 (i.e., active-MMP-2 when TNF $\alpha$ was added on day 7) and MMP-9 activity (when TNF $\alpha$ was added on day 2 and day 7) were seen compared to control (Fig. 6B and C). Interestingly, MMP-9 activity correlated with absence of tubules, whilst MMP-2 activity corresponds to the presence of tubules (Fig. 3A and B). Activation of MMP-2 (i.e., ratio between active over pro-form) was differ- ently regulated in co-cultures grown in EGM-2 medium or EGM-2 $\Theta$ compared to co-cultures treated with IL-6 or

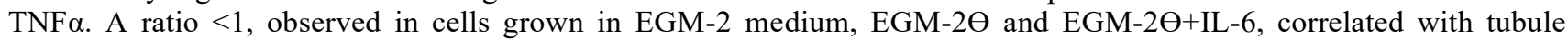
formation whereas a ratio $>1$ observed in cells grown in EGM-2 $\Theta+T N F \alpha$ resulted in little or no tubule formation. This result suggests that, according to the treatments investigated in this study, a correlation exists between tubule formation and MMP enzyme activity: tubules may partly be produced by modulating the activity of different MMP enzymes. Because it is possible MMP enzyme activity changed in function of total protein secretion which in turn could alter tubule formation, the concentration of total protein secreted into the medium (from HUVEC and NHDF cultured individually or in co-culture) was determined using the Bradford protein assay. There were, however, no differences in the concentration of total protein secreted between any of the test conditions and time points (data not shown).

A.

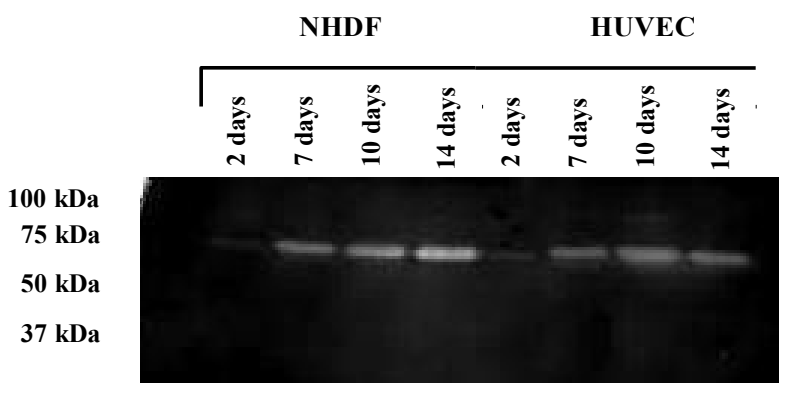

B.

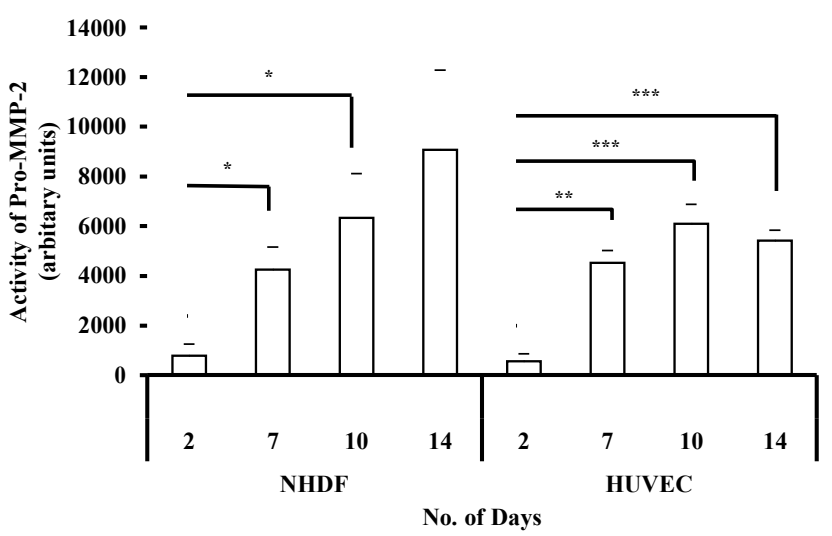

Fig. (5). (A) Pro-MMP-2 activity in NHDF and HUVEC cultured over 14 days. MMP enzymes released from cells into the medium on days 2 , 7, 10 and 14 was collected and analysed by gel zymography. Images presented are representative of 3 independent experiments. (B) Quantification of pro-MMP-2 activity using Image $\mathbf{J}$ software. Results are presented as mean \pm SEM of 3 independent experiments; $* P<0.05$, $* * P<0.01, * * * P<0.001$. 

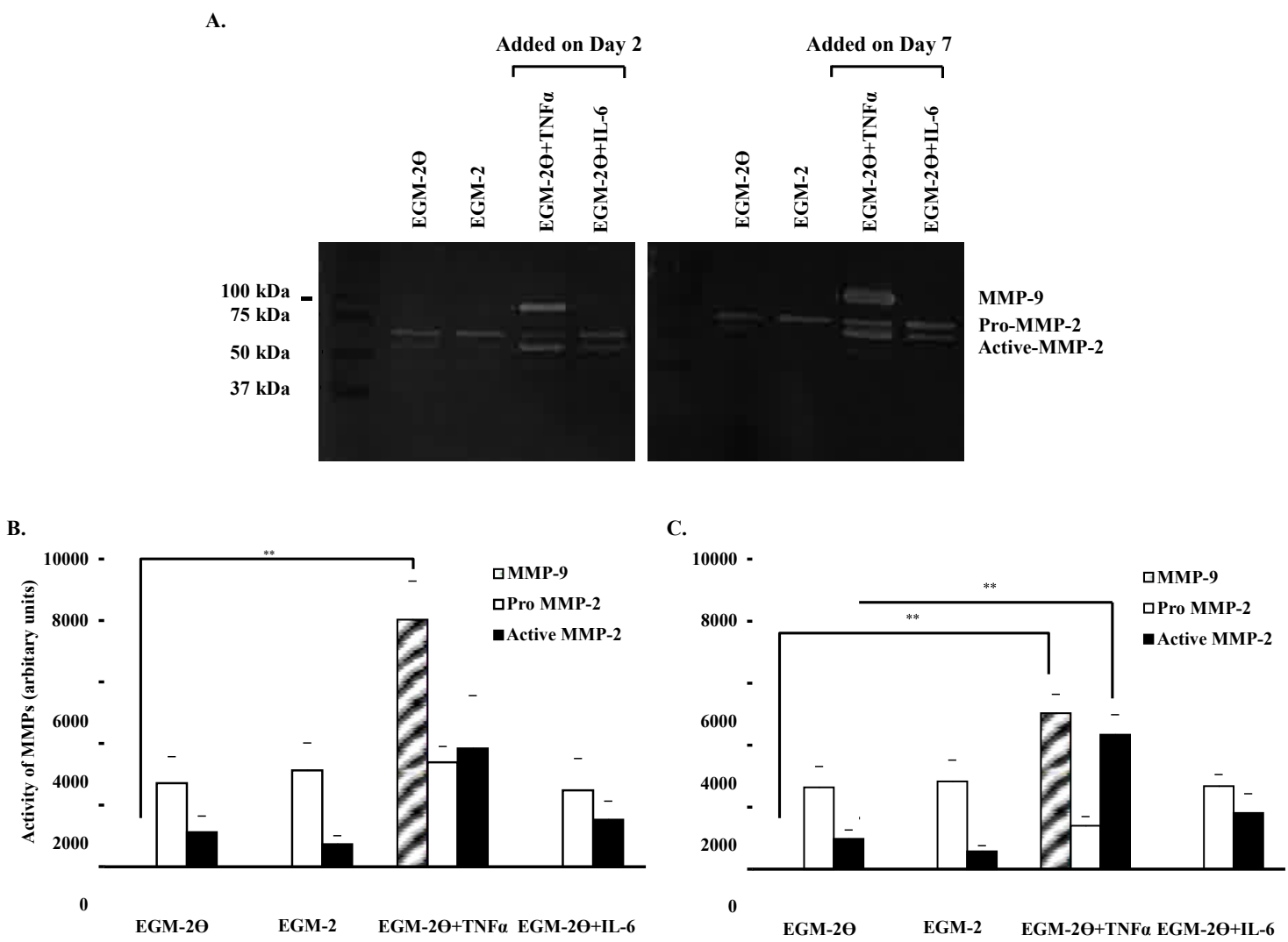

Fig. (6). (A) MMP-9, pro-MMP-2 and active-MMP-2 activity in HUVEC/NHDF co-cultures cultured, over 14 days, in EGM-2Ө, EGM-2 (positive control for tubule formation), EGM-2Ө+TNF $\alpha(10 \mathrm{ng} / \mathrm{mL})$ or EGM-2Ө+IL-6 (10 ng/mL). MMP enzymes released from the co- culture into the medium on day 14 was collected and analysed by gel zymography. Images presented are representative of 3 independent experiments. Quantification of MMP activity in medium with treatments (B) added on Day 2 and $(\mathbf{C})$ added on Day 7 using Image $\mathrm{J}$ soft- ware. Results are presented as mean \pm SEM of 3 independent experiments; $* P<0.05, * * P<0.01, * * * P<0.001$.

\section{DISCUSSION}

In this study, the effects of two inflammatory cytokines, TNF $\alpha$ and IL-6 were investigated. Experiments included assessing cell viability, tubule formation, secretion of VEGF, E-selectin and $\mathrm{PGE}_{2}$, and MMP enzyme activity using a more versatile in vitro HUVEC/NHDF co-culture system compared to the one developed by Bishop et al. [15]. This system has been established appositely and characterised (Fig. 2) to allow in depth studies of the role of specific molecules in modulating angiogenesis in addition to the heterogeneous cellular environment formed.

This study showed that both HUVEC and NHDF were more responsive to TNF $\alpha$ than IL-6; indicating that TNF $\alpha$ induced greater changes to cell viability than IL-6. Additionally, TNF $\alpha$ significantly decreased the formation of tubules. In contrast, and similar to the results observed in co-cultures grown in EGM-2 medium, IL-6 significantly increased tubule formation. TNF $\alpha$ completely prevented formation of tubules when added on day 2 and stopped any further development of tubule formation once the process had already started as evident when added on day 7. IL-6, on the contrary, promoted the formation of tubules when added on day 2 and increased tubule formation once the process had already started as evident when added on day 7 . These observations on tubule formation further indicate that angiogenesis is modulated by specific inflammatory markers. Additionally, this study has highlighted the importance of considering the time after tubule initiation when observing the generation and growth of endothelial tubules. This is the first time that a time course after initial tubule formation has been investigated and reported in the literature. Limited reports are available on the effect of TNF $\alpha$ or IL-6 alone in an in vitro co-culture cell system, except for a study conducted by Hashizume and colleagues [26]. Their study used fibroblast-like synovial cells from rheumatoid arthritis patients and HUVEC co-cultures to examine the effect of TNF $\alpha$ or IL- 6 on tubule formation over 21 days; neither TNF $\alpha$ nor IL-6 induced tubule formation. Hashi- zume and colleagues [26] measured the final outcome (i.e., tubule formation after 21 days co-culture) and did not differentiate between promoting/preventing or increasing/reducing angiogenesis which was examined in this study. Furthermore, secretion of key angio- genic/tumourigenic factors and re-modelling of ECM via MMP activity has not been fully investigated previously in relation to TNF $\alpha$ or IL-6. These factors have been investigated in more detail in this work in order to improve the understanding of the role of cytokines in modulating angiogenesis. 
It is well known that VEGF is one of the most important factors for accelerating angiogenesis [17]. In order to determine the relationship between tubule formation and release of VEGF, the concentration of VEGF secreted into conditioned medium was investigated using ELISA. Results revealed that co-culture treatment with TNF $\alpha$ induced an in- crease in secretion or accumulation of VEGF in the medium compared to co-cultures grown in EGM-2 $\Theta$ and the increase in concentration paralleled the lack of tubules formed. These observations support previous studies which emphasise the key role and requirement of VEGF in new tubule formation [27].

During inflammation, EC are activated by inflammatory cytokines, such as TNF $\alpha$, interleukin-1 (IL-1) and interferon $\gamma$ (IFN $\gamma$ ), which induce up-regulation of adhesion molecules expression including E-selectin [28]. E-selectin (CD62E) is an EC-specific adhesion molecule involved in initial vessel wall binding [29]. Although expression of E- selectin has been previously described in single-cell conditions [30], insight into the regulation of E-selectin expression and endothelial functioning following incubation with inflammatory cytokines remains, to the best of our knowledge, to be determined in an in vitro EC/fibroblast co- culture system.

Results in this study revealed no change in E-selectin expression, except for co-cultures grown in EGM- 2Ө+TNF $\alpha$. This may suggest that the time points selected for medium analysis (collected on days 2, 7 or 14 after initial establishment of the HUVEC/NHDF co-culture system) were not ideal to observe any changes in E-selectin expression with EGM-2, EGM-2Ө or EGM-2Ө+IL-6; especially since this is an early event in angiogenesis. Previous studies have shown timedependent up-regulation of E-selectin expression, observed by immunofluorescence, in HUVEC activated with TNF $\alpha$ (4 $\mathrm{ng} / \mathrm{mL}$ ) [28]. Griffioen and colleagues [28] also observed up-regulation of E-selectin ex- pression by IL-1 $\alpha$ and IFN $\gamma$. Similarly, Mason and colleagues [31] observed E-selectin up-regulation, via surface and mRNA expression, in HUVEC stimulated with $\mathrm{TNF} \alpha(10 \mathrm{ng} / \mathrm{mL})$. The results presented in this study have shown, for the first time, that cells grown in a co-culture system (HUVEC/NHDF) also increased E-selectin expression when stimulated with TNF $\alpha$. Also, as Eselectin mRNA expression is barely detectable in unstimulated HUVEC [31]; it is not unsurprising that E-selectin expression was only observed in co-cultures treated with EGM-2Ө+TNF $\alpha$ because TNF $\alpha$ itself will further induce inflammation. Furthermore, it has been shown that angiogenic growth factors (e.g., bFGF and VEGF) suppress inflammatory cytokine- induced up-regulation of E-selectin, as well as intercellular adhesion molecule-1 (ICAM-1) and vascular cell adhesion molecule-1 (VCAM-1) [28, 32]. In the experimental set-up used in this study, the EC/fibroblast co-culture system is grown in the recommended endothelial growth medium (EGM-2) which contains a mixture of growth factors, including bFGF and VEGF, which may influence the observed responses.

The protumourigenic prostanoid $\mathrm{PGE}_{2}$ is involved in promotion of angiogenesis $[33,34]$ and its production is known to

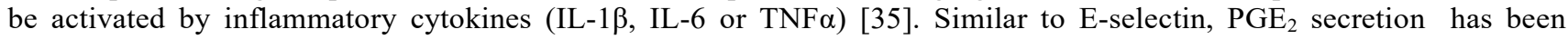
previously described in single-cell conditions in relation to angiogenesis [36], but, to our knowledge, its production remains to be determined in an in vitro EC/fibroblast co-culture system. Based on this information, our focus was driven by the assumption that the effect of TNF $\alpha$ or IL- 6 on tubule formation could be mediated by $\mathrm{PGE}_{2}$ production. Results from this study showed that the presence of tubules (incubation with EGM-2 medium and EGM-2Ө+IL-6) de- creased PGE 2 secretion, whilst the absence of tubules (incubation with EGM-2Ө+TNF $\alpha$ ) did not change $\mathrm{PGE}_{2}$ production compared to the control. Therefore, secretion of $\mathrm{PGE}_{2}$ was not evident in the medium collected on day 14 from the co-culture system. $\mathrm{PGE} 2$ is (i) rapidly released in response to inflammation [37], (ii) responsible for stimulation of EC migration and proliferation [38, 39] and, therefore, (iii) an important early event in angiogenesis, which confers that the time point selected for analysis of PGE $_{2}$ secretion (collected on day 14 after initial establishment of HUVEC/NHDF co- culture system) was limiting to observe any changes in production. However, the findings presented do give new insight into the role of $\mathrm{PGE}_{2}$ or lack thereof in tubule formation.

An initial step in the angiogenic process is degradation of the ECM [20, 21]. Following matrix breakdown, EC migrate and proliferate to form new vessels. Therefore, proteolytic enzyme activity, which degrades all components of the ECM, is essential for the initial process of angiogenesis and is performed by MMP enzymes [20, 21]. At present, regulation of MMP activity and the balance between MMP enzymes and their major inhibitors, Tissue Inhibitor of Metalloproteinases (TIMP) play a key role in EC migration and proliferation during angiogenesis [22, 40]. Given the important role of MMP enzymes in angiogenesis, the enzyme activity of MMP-2 and MMP-9 was measured in the media of HUVEC and NHDF individually and at the end point of co-culture incubation (day 14) in order to correlate enzyme activity to tubule formation using the widely accepted method of gel zymography. Untreated HUVEC and NHDF did not produce detectable MMP9. The pro-form of MMP-2 (72 kDa) was, however, visible in HUVEC and NHDF in this study. This data are in agreement with previous gel zymography studies reported in the literature: Jiang and colleagues [41] and Oak and colleagues [27] found only pro-MMP-2 expression in medium collected from cultured HUVEC, whilst Mauro and colleagues [42] detected the expression of pro-MMP-2 and MMP-9 in conditioned medium from cultured HUVEC. However, this was in contrast to what was observed in here, but the different medium used by Mauro et al. [42] could be responsible. Additionally, literature searches have shown little or no information is available for the enzyme activity of MMP-2 or MMP-9 in NHDF.

This study provides the first evidence that inflammatory markers can alter tubule formation in relation to the production and activity of MMP enzymes observed, at a final end point, in a heterogeneous cellular environment. Secretion of MMP-9 and activation of MMP-2 correlated with an absence of tubules, whilst the opposite was observed with the presence of tubules. In detail, treatment with TNF $\alpha$ resulted in MMP-9 production and activation of MMP-2 $(62 \mathrm{kDa})$. There is evidence to suggest that TNF $\alpha$ may be crucial in MMP production, in fact, it is known 
that TNF $\alpha(10 \mathrm{ng} / \mathrm{mL})$ stimulates MMP-9 expression via activation of ERK1/2 in HK-2 proximal tubular cells [43] or via activation of $\mathrm{NF \kappa B}$ in monocytes [44]. It remains to be seen whether these mechanisms are responsible for MMP9 production in this co-culture system and the investigation of the mechanisms by which MMP-9 expression is induced in the absence of tubule formation is currently on-going.

Additionally, the role of MMP enzymes in obesity associated inflammation still remains unclear, but a recent study by Miksztowicz and colleagues [45] found increased plasma activity of pro-MMP-2 in overweight/obese women (BMI 25$38.5 \mathrm{~kg} / \mathrm{m}^{2}, \mathrm{n}=26$ ) compared to normal weight women (BMI 18-24.9 kg/m², $\mathrm{n}=13$ ). Although these authors did not determine the origin of the measured pro-MMP-2, it is typically produced in most human tissue cell types, including adipose tissue. Interestingly, elevated levels of MMP-2 are associated with invasive behaviour [23] and findings reported here showed that pro-MMP-2 activity was higher than active-MMP-2 activity in co-cultures in the presence of tubules. Therefore, MMP-2 could be a key factor in angiogenesis and play an important role in tubule formation and cell-cell interactions.

The versatile co-culture model which utilised primary HUVEC co-cultured with primary NHDF was used to study the role of two cytokines, TNF $\alpha$ or IL-6, typically associ- ated with obese status in modulating new blood vessel formation in vitro. EC become organised amongst the fibro- blasts as corded structures which proliferate, thicken and lengthen through the cell matrix to form an intricate net- work of tubule structures over 14 days which are well established features [15]. Although studies have reported similar conclusions for TNF $\alpha$ [46-48] and IL-6 [49-51] in single cells to the one presented, the further development of the co-culture model by Bishop and colleagues [15] allowed a more versatile and flexible approach to investigate angiogenesis, and its progression, relative to single cell studies. This study has further characterised, from a technical point of view, this in vitro co-culture system especially in relation to manipulation of medium components and time of addition (after 2 days vs 7 days) of key inflammatory markers. Finally, this study has enabled us to reach a stage where the system can be manipulated according to specific requirements and allows a more detailed understanding of the role of inflammatory cytokines in the development of new blood vessels in direct relation to MMP activity and secretion of key angiogenic markers. Interestingly, the concentrations of cytokines used in the study have been shown by Bermano's group to play a role in the link between obesity and breast cancer [52] and this work will be taken forward in the context of obesity, breast cancer and angiogenesis ex vivo and in vivo.

\section{CONCLUSIONS}

In summary, this observational study showed treatment- specific differences in the regulation of angiogenesis and MMP activity. These findings show that it is critical to further improve understanding of how inflammatory cytokines can specifically contribute to the development of vascular complications in chronic inflammatory conditions such as the ones associated to obesity. In light of this, investigations in the effect of plasma from obese versus non-obese individuals using this versatile EC/fibroblast co-culture system are currently being explored.

\section{LIST OF ABBREVIATIONS}

$\begin{array}{lll}\text { EC } & = & \text { Endothelial cells Extracellular matrix } \\ \text { ECM } & = & \text { Human umbilical vein endothelial } \\ \text { HUVEC } & = & \text { cells } \\ \text { IL-6 } & = & \text { Interleukin-6 } \\ \text { MMP } & = & \text { Matrix metalloproteinases } \\ \text { NHDF } & = & \text { Pormal human dermal fibroblasts } \\ \text { PGE }_{2} & = & \text { Tumour necrosis factor- } \alpha \\ \mathrm{TNF} \alpha & = & \text { Vascular endothelial growth factor } \\ \text { VEGF } & \end{array}$

Coding for the different types of medium used: Endothelial basal medium (EBM-2) supplemented with SingleQuots (EGM-2 medium); EGM-2 medium minus the SingleQuot of VEGF (EGM-2Ө); EGM-2 medium minus the SingleQuot of VEGF supplemented with TNF $\alpha(10 \mathrm{ng} / \mathrm{mL})(\mathrm{EGM}-2 \mathrm{O}+\mathrm{TNF} \alpha)$; EGM-2 medium minus the SingleQuot of VEGF supplemented with IL-6 (10 ng/mL) (EGM-2Ө+IL-6).

\section{CONFLICT OF INTEREST}

The authors confirm that this article content has no conflict of interest.

Authors would like to thank TENOVUS Scotland and the Institute for Health and Wellbeing Research, Robert Gordon University for financial support. 


\section{ACKNOWLEDGEMENTS}

GAB conducted the HUVEC/NHDF co-culture experiments, collected and analysed data, wrote \& edited the manuscript. EB conducted and analysed the gel zymography experiments. MG and GB designed the study, wrote \& edited the manuscript. All authors read and approved the submitted version of the manuscript.

\section{REFERENCES}

[1] Olszanecka-Glinianowicz M, Zahorska-Markiewicz B, Janowska J, Zurakowski A. Serum concentration of nitric oxide, tumor necrosis factor (TNF)- $\alpha$ and TNF soluble receptors in women with over- weight and obesity. Metabolism 2004; 53: 1268-73.

[2] Hansen D, Dendale P, Beelen M, et al. Plasma adipokine and in- flammatory marker concentrations are altered in obese, as opposed to nonobese, type 2 diabetes patients. Eur J Appl Physiol 2010; 109: 379-404.

[3] Vistoropsky Y, Trofimov S, Malkin I, Kobyliansky E, Livshits G. Genetic and environmental determinants of hepatocyte growth fac- tor levels and their association with obesity and blood pressure. Ann Hum Biol 2008; 35: 93-103.

[4] Vona-Davis L, Rose DP. Adipokines as endocrine, paracrine, and autocrine factors in breast cancer risk and progression. EndocrRelat Cancer 2007; 14: 189-206.

[5] Aggarwal BB, Shishodia S, Sandur SK, Pandey MK, Sethi G. Inflammation and cancer: How hot is the link? Biochem Pharmacol 2006; 72 : $1605-21$.

[6] Dixon JB. The effect of obesity on health outcomes. Mol Cell Endocrinol 2010; 316: 104-8.

[7] Pandya NM, Dhalla NS, Santani DD. Angiogenesis - a new target for future therapy. Vasc Pharmacol 2006; 44:265-74.

[8] Bastelica D, Mavri A, Verdierl M, Berthet B, Juhan-Vague I, Alessi MC. Relationships between fibrinolytic and inflammatory parameters in human adipose tissue: strong contribution of TNF al- pha receptors to PAI-I levels. Thromb Haemost 2002; 88:481-7.

[9] Ghanim H, Aljada A. Hofmeyer D, Syed T, Mohanty P, Dandona P. Circulating mononuclear cells in the obese are in a proinflamma- tory state. Circulation 2004; 110: 1564-71.

[10] Nieman KM, Romero IL, Van Houten B, Lengyel E. Adipose tis- sue and adipocytes support tumorigenesis and metastasis. Biochim Biophys Acta 2013 Doi: 10.1016/j.bbalip.2013.02.010

[11] Alexandrakis MG, Tsirakis G. Circulating levels of soluble angio- genic factors in multiple myeloma: correlation with parameters of disease activity and prognosis. Curr Angiogen 2013; 2:76-95.

[12] Hanahan D, Folkman J. Patterns and emerging mechanisms of the angiogenic switch during tumourigenesis. Cell 1996; 86:353-64.

[13] Carmeliet P, Jain RK. Molecular mechanisms and clinical applica- tions of angiogenesis. Nature 2011; 473:298-307.

[14] Lijnen HR. Angiogenesis and Obesity. Cardiovasc Res 2008; 78: 286-93.

[15] Bishop ET, Bell GT, Bloor S, Broom IJ. An in vitro model of angi- ogenesis: basic features. Angiogenesis 1999; 3:335-44.

[16] Gupta MK, Qin RY. Mechanism and its regulation of tumor- induced angiogenesis. World J Gastroenterol 2003; 9: 1144-55.

[17] Christiaens V, Lijnen HR. Angiogenesis and development of adi- pose tissue. Mol Cell Endocrinol 2010; 318:2-9.

[18] Barbera-Guillem E, Nyhus JK, Wolford CC, Friece CR, Sampsel JW. Vascular Endothelial growth factor secretion by tumourinfiltrating macrophages essentially supports tumor angiogenesis, and IgG immune complexes potentiate the process. Cancer Res 2002; 62: 7042-9.

[19] Lee CC, Liu KJ, Huang TS. Tumor-associated macrophage: Its role in tumor angiogenesis. J Cancer Mol 2006; 2:135-40.

[20] Cornelius LA, Nehring LC, Roby JD, Parks WC, Welgus HG. Human dermal microvascular endothelial cells produce matrix metalloproteinases in response to angiogenic factors and migration. J Invest Dermatol 1995; 105: 170-6.

[21] Raza SL, Cornelius LA. Matrix metalloproteinases: Pro- and anti- angiogenic activities. J Invest Dermatol 2000; 5:47-54.

[22] Connelly L, Robinson-Benion C, Chont M, et al. A transgenic model reveals important roles for the NF- $\kappa \mathrm{B}$ alternative pathway (p100/p52) in mammary development and links to tumorigenesis. J Biol Chem 2007; 282: 10028-35.

[23] Van den Steen PE, Dubois B, Nelissen I, Rudd PM, Dwek RA, Opdenakker G. Biochemistry and molecular biology of gelatinase B or matrix metalloproteinase-9 (MMP-9). Crit Rev Biochem Mol 2002; 37: 375-536.

[24] Donovan D, Brown NJ, Bishop ET, Lewis CE. Comparison of three in vitro human 'angiogenesis' assays with capillaries formed in vivo. Angiogenesis 2001; 4: 113-21.

[25] Mosmann T. Rapid colorimetric assay for cellular growth and survival: application to proliferation and cytotoxicity assays. J Immunol Methods 1983; 65: 55-63.

[26] Hashizume M, Hayakawa N, Suzuki M, Mihara M. IL-6/IL-6R trans-signalling, but not TNF- $\alpha$ induced angiogenesis in an HU- VEC and synovial cell co-culture system. Rheumatol Int 2009; 29: 1449-54.

[27] Oak J-H, Nakagawa K, Oikawa S, Miyazawa T. Amadori- glycatedphosphatidylethanolamine induces angiogenic differentia- tions in cultured human umbilical vein endothelial cells. FEBS Lett 2003; 555: 419-23.

[28] Griffioen AW, Damen CA, Blijham GH, Groenewegen G. Tumour angiogenesis is accompanied by a decreased inflammatory re- sponse of tumour-associated endothelium. Blood 1996; 88:667-73.

[29] Rahman A, Kefer J, Bando M, Niles WD, Malik AB. E-selectin expression in human endothelial cells by TNF- $\alpha$-induced oxidant generation and NF-kB activation. Am J Phys 1998; 275: L533-44.

[30] Unger RE, Krump-Konvalinkova V, Peters K, Kirkpatrick CJ. In vitro expression of the endothelial phenotype: Comparative study of primary isolated cells and cell lines, including the novel cell line HPMEC-ST1.6R. Microvasc Res 2002; 64: 384-97.

[31] Mason JC, Yarwood H, Sugars K, Haskard DO. Human umbilical vein and dermal microvascular endothelial cells show heterogene- ity in response to PKC activation. Am J Phys 1997; 273: C1233-40.

[32] Zhang H, Issekutz AC. Down-modulation of monocyte transendo- thelial migration and endothelial adhesion molecule expression by fibroblast growth factor. Am J Pathol 2002; 160:2219-30.

[33] Form DM, Auerbach R. PGE 2 and angiogenesis. Proc Soc Exp Biol Med 1983; 172: 214-8.

[34] Díaz-Flores L, Gutierrez R, Valladares F, Varela H, Perez M. In- tense vascular sprouting from rat femoral vein induced by prosta- glandins $\mathrm{E}_{1}$ and $\mathrm{E}_{2}$. Anat Rec 1994; 238: 68-76.

[35] Bishop-Bailey D, Mitchell JA, Warner TD. COX-2 in cardiovascu- lar disease. Arterioscler Thromb Vasc Biol 2006; 26 : 956-8.

[36] Brecht K, Weigert A, Hu J, et al. Macrophages programmed by apoptotic cells promote angiogenesis via prostaglandin $\mathrm{E}_{2}$. FASEB J 2011; 25: $1-10$. 
[37] Kozak KR, Crews BC, Ray JL, Tai HH, Morrow JD, Marnett LJ. Metabolism of prostaglandin glycerol esters and prostaglandin ethanolamides in vitro and in vivo. J Biol Chem 2001; 276: 36993-8.

[38] Rao R, Redha R, Macias-Perez I, et al. Prostaglandin E2-EP4 re- ceptor promotes endothelial cell migration via ERK activation and angiogenesis in vivo. J Biol Chem 2007; 282: 16959-68.

[39] Finetti F, Solito R, Morbidelli L, Giachetti A, Ziche M, Donnini S. Prostaglandin $\mathrm{E}_{2}$ regulates angiogenesis via activation of fibroblast growth factor receptor-1. J Biol Chem 2008; 283:2139-46.

[40] Stamenkovic I. Extracellular matrix modelling: the role of matrix metalloproteinases. J Pathol 2003; 200:448-64.

[41] Jiang C, Ganther H, Lu J. Monomethyl selenium-specific inhibition of MMP-2 and VEGF expression: Implications for angiogenic switch regulation. Mol Carcinogen 2000; 29:236-50.

[42] Mauro A, Buscemi M, Gerbino A. Immunohistochemical and tran- scriptional expression of matrix metalloproteinases in full-term human umbilical cord and human umbilical vein endothelial cells. J Mol Histol 2010; 41: 367-77.

[43] Nee LE, McMorrow T, Campbell E, Slattery C, Ryan MP. TNF $\alpha$ and IL-1 $\beta$-mediated regulation of MMP-9 and TIMP-1 in renal proximal tubular cells. Kidney Int 2004; 66: 1376-86.

[44] Nguyen J, Goqusev J, Knapnougel P, Bauvois B. Protein tyrosine kinase and p38 MAP kinase pathways are involved in stimulation of matrix metalloproteinase-9 by TNF-alpha in human monocytes. Immunol Lett 2006; 106:34-41.

[45] Miksztowicz V, Siseles, N, Fernandez Machulsky N, Schreier L, Berg G. Increase in MMP-2 activity in overweight and obese women is associated with menopausal status. Climacteric 2012; 15: 602-6.

[46] Leibovich SJ, Polverini PJ, Shepard HM, Wiseman DM, Shively V, Nuseir N. Macrophage-induced angiogenesis is mediated by tu- mour necrosis factor- $\alpha$. Nature 1987; 329: 630-2.

[47] Koolwijk P, van Erck MGM, de Vree WJA, et al. Cooperative effect of TNF $\alpha$, bFGF, and VEGF on the formation of tubular structures of human microvascular endothelial cells in a fibrin ma- trix role of urokinase activity. J Cell Biol 1996; 132: 1177-88.

[48] Siemerink MJ, Klaassen I, Vogels IMC, Griffioen AW, van Noor- den CJF, Schlingemann RO. CD34 marks angiogenic tip cells in human vascular endothelial cell cultures. Angiogenesis 2012; 15: 151-63.

[49] Motro B, Itin A, Sachs L, Keshet E. Pattern of interleukin 6 gene expression in vivo suggests a role for this cytokine in angiogenesis. Proc Natl Acad Sci USA 1990; 87: 3092-6.

[50] Huang SP, Wu MS, Shun CT, et al. Interleukin-6 increases vascu- lar endothelial growth factor and angiogenesis in gastric carcinoma. J Biol Med Sci 2004; 11:517-27.

[51] Jee $\mathrm{SH}$, Chu CY, Chiu HC, et al. Interleukin-6 induced basic fibro- blast growth factor-dependent angiogenesis in basal cell carcinoma cell line via JAK/STAT3 and PI3-kinase/AKt pathways. J Invest Dermatol 2004; 123: 1169-75.

[52] Weichhaus M, Broom I, Bermano G. The molecular contribution of TNF- $\alpha$ in the link between obesity and breast cancer. Oncol Rep 2011; 25: 477-83.

Received: September 18, 2014 Revised: October 17, 2014 Accepted: November 12, 2014 